\title{
Recent Updates in the Endoscopic Diagnosis of Barrett's Oesophagus
}

\author{
Neel Sharma Khek Yu Ho \\ Asia Pacific Barrett's Consortium, National University Hospital Singapore, Singapore
}

\section{Key Words}

Barrett's oesophagus · Endoscopic diagnosis · Oesophageal adenocarcinoma

\begin{abstract}
Background: Barrett's oesophagus (BO) is a premalignant condition associated with the development of oesophageal adenocarcinoma (OAC). Despite the low risk of progression per annum, OAC is associated with significant morbidity and mortality, with an estimated 5-year survival of $10 \%$. Furthermore, the incidence of OAC continues to rise globally. Therefore, it is imperative to detect the premalignant phase of $\mathrm{BO}$ and follow up such patients accordingly. Summary: The mainstay diagnosis of BO is endoscopy and biopsy sampling. However, limitations with white light endoscopy (WLE) and undertaking biopsies have shifted the current focus towards real-time image analysis. Utilization of additional tools such as chromoendoscopy, narrow-band imaging (NBI), confocal laser endomicroscopy (CLE), and optical coherence tomography $(\mathrm{OCT})$ are proving beneficial. Furthermore, it is also becoming more apparent that often these tools are utilized by experts in the field. Therefore, for the non-expert, training in these systems is key. Currently as yet, the methodologies used for training optimization require further inquiry. Key Message: (1) Real-time imaging can serve to minimize excess biopsies. (2) Tools such as chromoendoscopy, NBI, CLE, and OCT can help to compliment WLE. Practical Implications: WLE is associated with limited sensitivity. Biopsy sampling is cost-ineffective and associated with sampling error. Hence, from a practical perspective, endoscopists should aim to utilize additional tools to help in real-time image interpretation and minimize an overreliance on histology.

(C) 2016 S. Karger AG, Basel
\end{abstract}

\section{Background}

Barrett's oesophagus (BO) is a premalignant condition associated with the development of oesophageal adenocarcinoma (OAC). Despite the low risk of progression per annum, OAC is associated with worsening morbidity and mortality, with an estimated 5-year survival of 
$10 \%$. Furthermore, the incidence of OAC continues to rise globally. Therefore, it is imperative to detect the premalignant phase of $\mathrm{BO}$ and follow up such patients accordingly.

This review highlights the recent updates in the endoscopic diagnosis of BO.

\section{Endoscopic Diagnosis}

The gold standard diagnosis of BO is white light endoscopy (WLE) and biopsy sampling as per the Seattle protocol. This involves biopsies at $1-2 \mathrm{~cm}$ in a quadrantic approach. However, WLE is associated with limited sensitivities. Furthermore, in view of the issues related to biopsy sampling errors, significant cost, and poor adherence to the Seattle protocol, there has been a push towards the use of enhanced imaging in the hope to eliminate the need for excessive biopsies as per the Preservation and Incorporation of Valuable Endoscopic Innovations (PIVI) initiative [1]. Numerous tools currently exist to further enhance diagnosis including chromoendoscopy, narrow-band imaging (NBI), confocal endomicroscopy, and optical coherence tomography (OCT) to name but a few. Thresholds recommended for such imaging comprise a sensitivity of $>90 \%$, a negative predictive value (NPV) of $>98 \%$, and a specificity of at least $80 \%$ [1].

The American Society for Gastrointestinal Endoscopy (ASGE) calculated the pooled sensitivity, NPV, and specificity for chromoendoscopy by using acetic acid and methylene blue, electronic chromoendoscopy by using NBI, and confocal laser endomicroscopy (CLE) for the detection of dysplasia. Results showed that the sensitivity, NPV, and specificity for acetic acid chromoendoscopy were 96.6\% [95\% confidence interval (CI), 95-98], 98.3\% (95\% CI, 94.899.4), and $84.6 \%$ (95\% CI, 68.5-93.2), respectively. The sensitivity, NPV, and specificity for electronic chromoendoscopy by using NBI were $94.2 \%$ (95\% CI, 82.6-98.2), 97.5\% (95\% CI, 95.1-98.7), and 94.4\% (95\% CI, 80.5-98.6), respectively. The sensitivity, NPV, and specificity for CLE were 90.4\% (95\% CI, 71.9-97.2), 98.3\% (95\% CI, 94.2-99.5), and 92.7\% (95\% CI, 87-96), respectively [2].

A further diagnostic study was performed focusing on the difference in yield for the detection of dysplasia or cancer using advanced imaging versus WLE. Overall, advanced imaging techniques increased the diagnostic yield for the detection of dysplasia or cancer by $34 \%$ (95\% CI, 20-56; $\mathrm{p}<0.0001$ ). Additional analysis showed that virtual chromoendoscopy significantly increased the diagnostic yield (risk difference, $0.34 ; 95 \% \mathrm{CI}, 0.14-0.56 ; \mathrm{p}<$ 0.0001 ). The risk difference for chromoendoscopy was 0.35 ( $95 \% \mathrm{CI}, 0.13-0.56 ; \mathrm{p}=0.0001$ ). There was no significant difference between virtual chromoendoscopy and chromoendoscopy $(\mathrm{p}=0.45)[3]$.

With regard to NBI, the Barrett's International NBI Group (BING), composed of NBI experts from the United States, Europe, and Japan, developed a validated NBI classification system for identifying dysplasia and cancer in BO. Sixty NBI images of non-dysplastic BO, high-grade dysplasia (HGD), and OAC were reviewed to characterize mucosal and vascular patterns visible by NBI. Patients undergoing surveillance or endoscopic treatment for BO were recruited and high-quality NBI images obtained with histological analysis of biopsies. Experts individually reviewed $50 \mathrm{NBI}$ images to validate the BING criteria and then evaluated 120 additional NBI images (not previously viewed) to determine whether the criteria accurately predicted the histology results. The BING criteria identified patients with dysplasia with an $85 \%$ overall accuracy, $80 \%$ sensitivity, $88 \%$ specificity, $81 \%$ positive predictive value (PPV), and 88\% NPV. When dysplasia was identified with a high level of confidence, these values were $92,91,93,89$, and 95\%, respectively. The overall strength of interobserver agreement was substantial $(\kappa=0.681)$ [4]. 
In relation to the PIVI initiative and a call for more image-guided diagnosis, Appannagari et al. [5] analysed insights from 172 gastroenterologists. 140 (81.4\%) stated they would implement the PIVI recommendations into practice. The two main barriers to the implementation of the protocol were medical-legal and financial reasons. Of the 32 gastroenterologists who were not willing to implement the imaging-guided surveillance protocol, $20(62.5 \%)$ stated that they would implement it if there were a financial incentive.

Magnification endoscopy is also a worthwhile tool for diagnostic use. A prospective study with 28 endoscopists from 11 countries (Asia-Pacific region) took part as assessors in diagnosing BO. In total, 35 patients were assessed using 150 slides from WLE-z (z magnification) and NBI-z randomly arranged. Results noted that the overall accuracy for WLE-z and NBI-z images was 87.1 and $88.7 \%$, respectively. When images from the two modalities were placed side by side, the accuracy increased to $90.3 \%$. The sensitivity, specificity, PPV, and NPV of WLE-z were $48,92,45$, and $93 \%$ while with NBI-z, these improved to $89,89,56$, and $98 \%$, respectively. When both imaging modalities were viewed together, they improved further to 93, 90, 61, and 99\% [6].

In an analysis of WLE, NBI, and autofluorescence imaging for the detection of HGD/OAC, autofluorescence imaging alone had a sensitivity, specificity, and NPV of 50,61, and 71\%, respectively, with an overall accuracy of $57 \%$. By using magnification NBI in tandem fashion, the sensitivity and NPV improved to 71 and $76 \%$, respectively, with a decrease in specificity to $46 \%$ and in overall accuracy to $55 \%$. Moderate interobserver agreement for surface patterns and prediction of histology was noted [7].

A randomized crossover trial compared high-definition WLE (HD-WLE) and NBI for the detection of intestinal metaplasia (IM) and neoplasia in 123 BO patients. Both HD-WLE and NBI detected 104/113 (92\%) patients with IM, with NBI requiring fewer biopsies per patient ( 3.6 vs. 7.6; $\mathrm{p}<0.0001$ ). NBI detected a higher proportion of areas with dysplasia (30 vs. 21\%; $\mathrm{p}=0.01$ ). During examination with NBI, all areas of HGD and cancer had an irregular mucosal or vascular pattern [8].

With respect to confocal endomicroscopy, an analysis of its worth has recently been determined through expert consensus. Initial statements were developed by 5 group leaders, based on the available clinical evidence. These statements were then voted on and edited by 26 participants, using a modified Delphi approach. After two rounds of voting, statements were validated if the threshold of agreement was higher than 75\%. Agreement for CLE use in $\mathrm{BO}$ was reached with regard to evaluation, in combination with red flag techniques, e.g., chromoendoscopy, for patients with dysplasia initially identified with electronic enhancement, for dysplasia identified by endoscopic surveillance, for distinguishing cardia from IM based on goblet cell existence, its superior value over WLE for the diagnosis of IM, its use in reducing the need for a biopsy if CLE proved negative during random sampling, improvements in the yield of neoplasia compared with standard WLE and random biopsies, in combination with WLE for the detection of dysplasia, for therapeutic intervention in cases of positive CLE in a neoplastic appearing oesophagus, for location definition and lateral neoplasia extent prior to therapy, and as a useful tool for increased diagnostic yield in surveillance guidelines [9].

Changes are also taking place with regard to the use of OCT. A recent evaluation study of the safety and feasibility of the NvisionVLE system was performed in a prospective multicentre study. This involved 100 patients with suspected BO, including 52 patients with prior endotherapy. The NvisionVLE Imaging System, a rotating optical probe, provided images of the mucosa and submucosa through a 6 -cm segment length and a $360^{\circ}$ scan of the distal oesophagus. VLE imaging was successfully performed in 87 cases. After VLE imaging, biopsy specimens were obtained and mucosal resection was performed in 20 patients. The final pathological diagnoses of the patients assessed were adenocarcinoma (4 patients), HGD (10 patients), low-grade dysplasia (LGD; 11 patients), indefinite (5 patients), IM (29 patients), 
and normal squamous cells (18 patients). VLE was not completed in 13 of 100 patients (13\%) because of optical probe and console limitations. There were 2 minor adverse events, namely mucosal lacerations, not requiring intervention [10].

A further study by Hatta et al. [11] assessed the use of probe-type OCT imaging to evaluate Barrett's mucosa buried underneath oesophageal squamous epithelium. A single-centre prospective study was undertaken of patients with Barrett's adenocarcinoma from 2008 to 2014. The enrolled patients were examined by a probe-type OCT in vivo, followed by en bloc endoscopic submucosal dissection. The overall accuracy, sensitivity, specificity, PPV, and NPV of the buried mucosa in the OCT imaging were $85.7 \%$ (12/14), 77.8\% (7/9), 100\% (5/5), $100 \%$ (7/7), and $71.4 \%(5 / 7)$, respectively. OCT, however, could not easily distinguish nondysplastic glands from dysplastic glands [11].

Utilization of end oscopic ultrasound (EUS) has been assessed from a Barrett's perspective. Qumseya et al. [12] analysed 1,278 articles, of which 11 met the inclusion criteria, comprising a total of 656 patients. Based on a random-effects model, the proportion of patients with advanced disease (dysplasia/OAC) detected on EUS was 14\% (95\% CI, 8-22; p < 0.0001). In further analysis, the pooled proportion of patients with advanced disease on EUS in the absence of nodules was 4\% (95\% CI, 2-6; p < 0.0001).

Although the primary focus of this review is endoscopy based, pathology-based diagnosis enhancement is worthy of mention. One of the drawbacks in this regard is the limited interobserver agreement for Barrett's related dysplasia. Wide area transepithelial sampling is a technique focused on wider area sampling of BO tissue followed by computer-assisted analysis. Vennalaganti et al. [13] randomly selected wide area transepithelial sampling slides with varying degrees of BO dysplasia for a review by 4 pathologists. Each pathologist graded the slides as non-dysplastic, LGD, or HGD/OAC. $149 \mathrm{BO}$ slides were evaluated in a blinded manner. The slides included the following: no dysplasia ( $n=109)$, LGD, and HGD/OAC $(n=40)$. The overall mean $\kappa$ value for all 3 diagnoses for the 4 observers was calculated at 0.86 (95\% CI, $0.75-0.97)$. The $\kappa$ values (95\% CI) for HGD/OAC, indefinite for dysplasia/LGD, and no dysplasia were 0.95 (0.88-0.99), 0.74 (0.61-0.85), and 0.88 (0.81-0.94), respectively.

From a training perspective, research is underway to help determine how best to educate learners in Barrett's related image diagnosis. One such study focused on CLE, involving participants with no prior CLE experience randomized to either in-class didactic (group 1) or selfdirected teaching groups (group 2) using audio PowerPoint. After initial training, all participants graded an initial set of 20 CLE videos and reviewed responses with an expert. Finally, all participants completed interpretations of a further 40 videos. Overall diagnostic accuracy for neoplasia prediction by CLE was 77\% (95\% CI, 74.0-79.2); of predictions made with high confidence (53\%), the accuracy was 85\% (95\% CI, 81.8-87.8). The overall accuracy and interobserver agreement was significantly higher in group 1 than in group 2 for all predictions ( 80.4 vs. $73 \%$; $p=0.005$ ) and for high-confidence predictions ( 90 vs. $80 \% ; p<0.001$ ). Following feedback (after the initial 20 videos), the overall accuracy improved from 73 to $79 \%(p=0.04)$, driven by a significant improvement in group 1 ( 74 to $84 \% ; p<0.01)$. Accuracy of prediction significantly improved with time in endoscopy training ( $p=0.003$ ) [14].

A further randomized controlled trial on training focused on NBI. Half of the participants were taught in a classroom setting by an endoscopist with expertise in NBI, whereas the other participants were in a self-directed group that received an automated version of the presentation with audio commentary. Participants completed a test of 40 randomized NBI images, predicting the histology and indicating their confidence levels in the diagnosis. Results noted no difference in accuracy between the in-classroom and self-directed groups (57.5 vs. $57.2 \%$; $\mathrm{p}=1.0$ ). The in-classroom group had a significantly higher percentage of high-confidence answers (57.2 vs. $41.1 \%$; $\mathrm{p} \leq 0.01$ ), but there was no significant difference in accuracy (60.7 vs. $66.4 \% ; p=0.34)[15]$. 


\section{Closing Remarks}

The mainstay diagnosis of BO is endoscopy and biopsy sampling. However, limitations with WLE and undertaking a biopsy has shifted the current focus towards real-time image analysis. Utilization of additional tools such as chromoendoscopy, NBI, CLE, and OCT are proving beneficial as per the recent studies highlighted here. Furthermore, what is also becoming apparent is that often these tools are utilized by experts in the field. Therefore, for the non-expert, training in these systems is key. Currently as yet, the methodologies used for training optimization require further inquiry.

\section{Disclosure Statement}

No conflicts of interest exist.

\section{References}

1 Sharma P, Savides TJ, Canto MI, Corley DA, Falk GW, Goldblum JR, et al: The American Society for Gastrointestinal Endoscopy PIVI (Preservation and Incorporation of Valuable Endoscopic Innovations) on imaging in Barrett's esophagus. Gastrointest Endosc 2012;76:252-254.

$\longrightarrow 2$ Thosani N, Abu Dayyeh BK, Sharma P, Aslanian HR, Enestvedt BK, Komanduri S, et al: ASGE Technology Committee systematic review and meta-analysis assessing the ASGE Preservation and Incorporation of Valuable Endoscopic Innovations thresholds for adopting real-time imaging-assisted endoscopic targeted biopsy during endoscopic surveillance of Barrett's esophagus. Gastrointest Endosc 2016;83:684-698.

-3 Qumseya BJ, Wang H, Badie N, Uzomba RN, Parasa S, White DL, et al: Advanced imaging technologies increase detection of dysplasia and neoplasia in patients with Barrett's esophagus: a meta-analysis and systematic review. Clin Gastroenterol Hepatol 2013;11:1562-1570.e1-e2.

4 Sharma P, Bergman JJ, Goda K, Kato M, Messmann H, Alsop BR, et al: Development and validation of a classification system to identify high-grade dysplasia and esophageal adenocarcinoma in Barrett's esophagus using narrow-band imaging. Gastroenterology 2016;150:591-598.

5 Appannagari A, Soudagar AS, Pietrzak C, Sharma P, Gupta N: Are gastroenterologists willing to implement imagingguided surveillance for Barrett's esophagus? Results from a national survey. Endosc Int Open 2015;3:E181-E185.

-6 Singh R, Jayanna M, Wong J, Lim LG, Zhang J, Lv J, et al: Narrow-band imaging and white-light endoscopy with optical magnification in the diagnosis of dysplasia in Barrett's esophagus: results of the Asia-Pacific Barrett's Consortium. Endosc Int Open 2015;3:E14-E18.

7 Giacchino M, Bansal A, Kim RE, Singh V, Hall SB, Singh M, et al: Clinical utility and interobserver agreement of autofluorescence imaging and magnification narrow-band imaging for the evaluation of Barrett's esophagus: a prospective tandem study. Gastrointest Endosc 2013;77:711-718.

-8 Sharma P, Hawes RH, Bansal A, Gupta N, Curvers W, Rastogi A, et al: Standard endoscopy with random biopsies versus narrow band imaging targeted biopsies in Barrett's oesophagus: a prospective, international, randomised controlled trial. Gut 2013;62:15-21.

-9 Wang KK, Carr-Locke DL, Singh SK, Neumann H, Bertani H, Galmiche JP, et al: Use of probe-based confocal laser endomicroscopy (pCLE) in gastrointestinal applications. A consensus report based on clinical evidence. United European Gastroenterol J 2015;3:230-254.

10 Wolfsen HC, Sharma P, Wallace MB, Leggett C, Tearney G, Wang KK: Safety and feasibility of volumetric laser endomicroscopy in patients with Barrett's esophagus (with videos). Gastrointest Endosc 2015;82:631-640.

11 Hatta W, Uno K, Koike T, Ara N, Asano N, Iijima K, et al: Feasibility of optical coherence tomography for the evaluation of Barrett's mucosa buried underneath esophageal squamous epithelium. Dig Endosc 2015, Epub ahead of print.

12 Qumseya BJ, Brown J, Abraham M, White D, Wolfsen H, Gupta N, et al: Diagnostic performance of EUS in predicting advanced cancer among patients with Barrett's esophagus and high-grade dysplasia/early adenocarcinoma: systematic review and meta-analysis. Gastrointest Endosc 2015;81:865-874.e2.

13 Vennalaganti PR, Naag Kanakadandi V, Gross SA, Parasa S, Wang KK, Gupta N, et al: Inter-observer agreement among pathologists using wide-area transepithelial sampling with computer-assisted analysis in patients with Barrett's esophagus. Am J Gastroenterol 2015;110:1257-1260.

$>14$ Rzouq F, Vennalaganti P, Pakseresht K, Kanakadandi V, Parasa S, Mathur SC, et al: In-class didactic versus selfdirected teaching of the probe-based confocal laser endomicroscopy (pCLE) criteria for Barrett's esophagus. Endoscopy 2016;48:123-127.

15 Daly C, Vennalaganti P, Soudagar S, Hornung B, Sharma P, Gupta N: Randomized controlled trial of self-directed versus in-classroom teaching of narrow-band imaging for diagnosis of Barrett's esophagus-associated neoplasia. Gastrointest Endosc 2016;83:101-106. 